\title{
Tunable zero-field Kondo splitting in a quantum dot
}

\author{
M. Larsson, ${ }^{1, *}$ J. S. Lim, ${ }^{2}$ R. López, ${ }^{2,3}$ and H. Q. Xu ${ }^{1,4}$ \\ ${ }^{1}$ Division of Solid State Physics, Lund University, P. O. Box 118, S-221 00 Lund, Sweden \\ ${ }^{2}$ Institut de Física Interdisciplinària i de Sistemes Complexos IFISC (CSIC-UIB), E-07122 Palma de Mallorca, Spain \\ ${ }^{3}$ Departament de Física, Universitat de les Illes Balears, E-07122 Palma de Mallorca, Spain \\ ${ }^{4}$ Department of Electronics and Key Laboratory for the Physics and Chemistry of Nanodevices, Peking University, Beijing 100871, China
}

(Received 14 February 2013; revised manuscript received 10 July 2013; published 7 August 2013)

\begin{abstract}
We present detailed low-temperature transport measurements of a single quantum dot formed in an InGaAs/InP heterostructure with a strong tunnel coupling to the source and drain leads. The conventional spin-1/2 Kondo effect is observed for the quantum dot in the $N=9$ charge state. By changing the voltages applied to the quantum dot barrier gates, we find a zero-field splitting of the Kondo resonance and a zero-bias differential conductance, which shows a nonmonotonic in-plane magnetic field and temperature dependence. Using a two-site Hubbard model, we show that the main observed features can be explained in terms of Kondo correlation effects resulting from the exchange interaction between two localized spins.
\end{abstract}

DOI: 10.1103/PhysRevB.88.085407

PACS number(s): 73.63.Kv, 75.75.-c

\section{INTRODUCTION}

In recent years quantum dots made from semiconductor materials have received much attention due to their potential as building blocks for spintronic and quantum computing devices. ${ }^{1}$ To realize these devices, studies of spin transport and spin coherence in semiconductor quantum dots are of great importance. One manifestation of a coherent spin state is the so-called Kondo effect, which in a quantum dot gives rise to an enhancement of the differential conductance at zero bias for temperatures below the characteristic Kondo temperature, $T_{K} \cdot{ }^{2,3}$ The conductance enhancement can be attributed to the formation of a coherent many-body state formed by electron spins on the leads and a single unpaired spin on the quantum dot. This conventional effect, usually referred to as the spin-1/2 Kondo effect, is expected to occur for an odd number of electrons on the dot and has been extensively studied in various quantum dot systems. ${ }^{4-11}$ In addition to the spin-1/2 Kondo effect, the large tunability of these systems has allowed for studies of different variants of the Kondo effect, such as Kondo correlation effects at singlet-triplet transitions for an even number of electrons on the dot, ${ }^{6,12-14}$ the so-called orbital Kondo effect, ${ }^{15}$ and the two-channel Kondo effect. ${ }^{16}$

Moreover, the exchange coupling between a quantum dot and a nearby impurity, the so-called two-impurity Kondo effect, has attracted interest. ${ }^{17-24}$ Controlled coupling to intentional impurities has been achieved experimentally ${ }^{17,18}$ and can be understood in terms of the so-called RudermanKittel-Kasuya-Yosida (RKKY) interaction. ${ }^{25,26}$ However, the coupling to unintentional impurities is still not fully understood and further work is needed in order to gain knowledge of the origin of the impurities and the nature of the interaction. ${ }^{22-24}$

In this work we present detailed results from a study of the conventional spin-1/2 Kondo effect and a gate-induced splitting of the same Kondo resonance in a lateral quantum dot with an odd occupation number at zero magnetic field. Zero-field splittings of Kondo resonances in single quantum dots have previously been reported in GaAs-based devices with intentional impurities ${ }^{17,18}$ and in carbon nanotube quantum dots where the splittings were attributed to interactions with unintentional magnetic impurities. ${ }^{22-24}$ A zero-field splitting of the Kondo resonance has also been reported for an evenoccupation quantum dot with a total spin $S=1,{ }^{27}$ where the splitting was attributed to a singlet-triplet transition and an associated two-stage Kondo process in the presence of single channel leads. ${ }^{12,28,29}$ We investigate Kondo correlation effects at the $N=9$ charge state in our quantum dot and find that the zero-bias Kondo resonance associated with the spin-1/2 Kondo effect can be split into two peaks by changing the voltages applied to the barrier gates. The single Kondo resonance is restored when a finite magnetic field is applied. We theoretically model our system using a two-site Hubbard model and show that the Kondo restoration can be described by a two-impurity Kondo effect scenario. Additionally, the observed nonmonotonic temperature dependence of the linear response conductance is in agreement with theoretical predictions. $^{20}$

\section{EXPERIMENTAL TECHNIQUES}

The quantum dot device was fabricated in a modulationdoped $\mathrm{In}_{0.75} \mathrm{Ga}_{0.25} \mathrm{As} / \mathrm{InP}$ heterostructure containing a twodimensional electron gas (2DEG) in a 9-mm-thick InGaAs quantum well layer buried $40 \mathrm{~nm}$ below the surface. A 700-nm-long and 150-nm-wide quantum wire was defined in the 2DEG by wet chemical etching together with a quantum point contact (QPC) defined in close proximity to the quantum wire. A $20-n m$ thin film of $\mathrm{HfO}_{2}$ was deposited locally on top of the device acting as a gate dielectric. Five local finger Ti/Au gates were fabricated over the quantum wire and one gate was fabricated on top of the QPC; ${ }^{30}$ see Fig. 1(a).

Transport measurements were performed in a dilution refrigerator with a dc bias applied in a symmetric fashion to both the source and the drain contacts. All measurements were carried out at a refrigerated temperature of about $30 \mathrm{mK}$, corresponding to an electron temperature of about $100 \mathrm{mK}$ unless otherwise noted. The local top gates $\lg 3$ and $\lg 5$ seen in Fig. 1(a) were used to define a single quantum dot in the quantum wire. The local gate $\lg 4$ was used to tune the dot occupation, whereas local gates $\lg 1$ and $\lg 2$ were grounded. The QPC gate lqpc was used to tune the conductance of the 


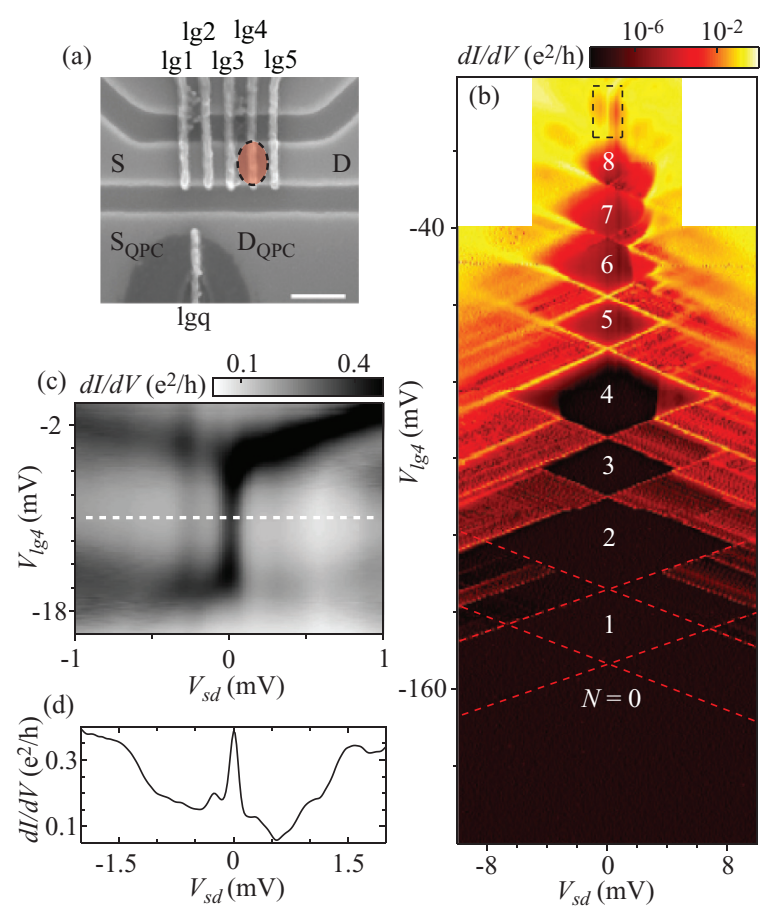

FIG. 1. (Color online) (a) Scanning electron micrograph of the measured device. A quantum dot is formed between gates $\lg 3$ and $\lg 5$. The QPC acts as a sensor for the charge states of the dot. The scale bar is $200 \mathrm{~nm}$. (b) Differential conductance on a logarithmic color scale measured as a function of source-drain voltage $V_{s d}$ and gate voltage $V_{l g 4}$. The total number of electrons $N$ in the dot is indicated. The $N=9$ Coulomb diamond shows a conductance peak at zero bias. Borders corresponding to the $N=1$ Coulomb diamond have been outlined with dashed red lines for clarity. (c) Close-up of the $N=9$ Coulomb diamond on a linear color scale showing increased conductance along zero bias. (d) Trace along the white dashed line, $V_{l g 4}=-10 \mathrm{mV}$, in (c).

QPC to allow sensitive charge sensing of the quantum dot charge state. ${ }^{30}$

\section{SPIN-1/2 KONDO EFFECT}

Figure 1(b) shows the differential conductance $d I / d V_{s d}$ through the quantum dot as a function of bias voltage $V_{s d}$ and gate voltage $V_{l g 4}$ using voltages $V_{l g 3}=-250 \mathrm{mV}$ and $V_{l g 5}=-415 \mathrm{mV}$ applied to the barrier gates. The total number of electrons, $N$, on the dot was verified by looking for sudden jumps in the conductance through the QPC, corresponding to changes in the charge state on the dot. The total number of electrons is indicated by the number inside each Coulomb diamond in the figure. For $V_{l g}<-153 \mathrm{mV}$ no further changes in the charge state of the quantum dot could be detected by the QPC (not shown here), indicating an empty dot. A conductance enhancement along zero bias is seen inside the $N=9$ Coulomb diamond, a feature which is consistent with the spin-1/2 Kondo effect in a quantum dot containing a spinunpaired electron. ${ }^{4-11}$ Figure 1(c) shows an enlarged plot of the $N=9$ Coulomb diamond, where the quantum dot is in the strong-coupling regime, with the zero-bias Kondo resonance clearly visible inside the Coulomb blockaded region [see also Fig. 1(d)], which shows a line trace along the white dashed line in Fig. 1(c). Neighboring even-occupation Coulomb diamonds do not show the zero-bias Kondo resonance, which suggests a spin-filling sequence of spin-up and spin-down electrons added consecutively to the dot, where only the odd-occupation Coulomb diamonds have a nonzero total spin. Following recent work, ${ }^{31}$ we estimate the Kondo temperature $T_{K} \sim 0.4 \mathrm{~K}$ from the full width at half maximum (FWHM) of $\sim 120 \mu \mathrm{V}$ of the Kondo resonance in Fig. 1(d) using the expression FWHM $=2 \sqrt{\pi} k_{B} T_{K}$. The Kondo temperature can also be estimated from the tunnel coupling, which can be determined from the linear response conductance:

$$
T_{K} \sim \frac{\sqrt{h \Gamma U}}{2 k_{B}} e^{-\pi U / 4 \Gamma h},
$$

where $U$ is the on-site Coulomb energy and $\Gamma=\Gamma_{S}+\Gamma_{D}$ is the sum of the tunnel rates to the source and drain leads. ${ }^{32}$ Note that Eq. (1) is only valid in the center of the Coulomb blockade region. The Coulomb energy $U=3.0 \mathrm{meV}$ is determined from half the width of the $N=9$ Coulomb diamond, whereas $\Gamma$ is determined from line shape fits of the two Coulomb blockade peaks on either side of the $N=9$ Coulomb blockade valley at $T=700 \mathrm{mK}\left(k_{B} T=60 \mu \mathrm{eV}\right)$ using the Breit-Wigner formula $^{33}$ (not shown). The evaluated tunnel coupling $h \Gamma \sim$ $1.3 \mathrm{meV}\left(h \Gamma \gg k_{B} T\right)$ satisfies the necessary condition to observe the Kondo effect, $h \Gamma>k_{B} T_{K}$, in our sample. We calculate $T_{K} \sim 1.8 \mathrm{~K}$ using the extracted values of $U$ and $\Gamma$ from Eq. (1). This Kondo temperature is several times larger than the value determined from the Kondo resonance width. This discrepancy is most likely explained by uncertainty in the determination of $\Gamma$.

Figure 2(a) shows the $N=9$ Coulomb diamond with a magnetic field $B=2 \mathrm{~T}$ applied in the 2DEG plane in the direction along the wire. The zero-bias Kondo resonance is split into two peaks due to lifting of the spin degeneracy at a finite magnetic field, with the peak positions given by the Zeeman energy $e V_{s d}= \pm g_{5}^{*} \mu_{B} B$, where $g_{5}^{*}$ is the effective $g$ factor of the fifth single-particle level in the dot. Based on the splitting of the Kondo resonance we extract an effective $g$ factor $\left|g_{5}^{*}\right| \sim 2.6$, which is similar to what has previously been found in few-electron InGaAs quantum dots. ${ }^{30,34}$

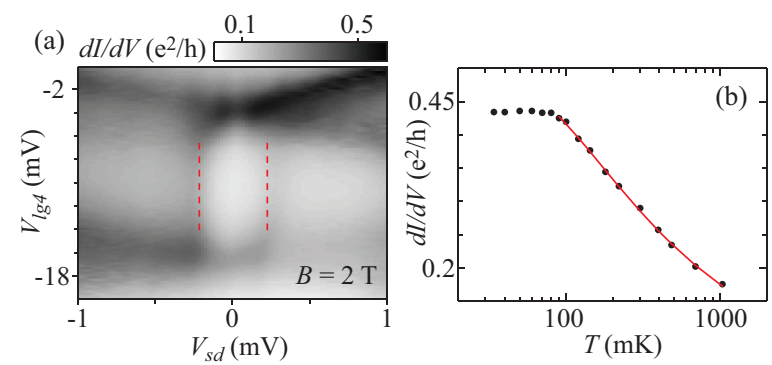

FIG. 2. (Color online) (a) Charge stability diagram of the $N=9$ Coulomb diamond with a magnetic field $B=2 \mathrm{~T}$ applied in the 2DEG plane showing Zeeman splitting of the Kondo resonance. The Zeeman split peaks are highlighted by dashed red lines. (b) Temperature dependence of the zero-bias differential conductance on a logarithmic temperature scale taken in the middle of the Coulomb diamond, $V_{l g 4}=-10 \mathrm{mV}$. The solid red line denotes a fit to the experimental data using the phenomenological expression given in Eq. (2). 
Figure 2(b) shows the temperature dependence on a logarithmic temperature scale of the differential conductance at zero bias for $V_{l g 4}=-10 \mathrm{mV}$ corresponding to the Kondo resonance in the $N=9$ Coulomb blockade region. The logarithmic temperature decay of the conductance for $T>100 \mathrm{mK}$ is further evidence that the zero-bias conductance peak stems from the spin- $1 / 2$ Kondo effect. The flat temperature response for $T<100 \mathrm{mK}$ is most likely due to saturation of the electron temperature in the sample. Furthermore, we estimate the Kondo temperature $T_{K} \sim 0.4 \mathrm{~K}$ by fitting the temperature dependence with the phenomenological expression ${ }^{35}$

$$
G(T)=G_{0} /\left[1+\left(2^{1 / s}-1\right)\left(T / T_{K}\right)^{2}\right]^{s},
$$

where we have used $G_{0}=0.51$ and $s=0.21$; see the red solid line in Fig. 2(b). The fact that the Kondo temperatures determined from the Kondo resonance FWHM and the temperature dependence agree well with each other suggests that the tunnel coupling $h \Gamma$ is overestimated as the Kondo temperature determined from Eq. (1) is significantly larger compared to the other estimations.

\section{TWO-IMPURITY KONDO EFFECT}

We now present measurements where the barrier gate voltages have been slightly changed to $V_{l g 3}=-260 \mathrm{mV}$ and $V_{l g 5}=-405 \mathrm{mV}$. As well as affecting the tunnel barriers and the lateral shape of the quantum dot, the decreased barrier gate voltage asymmetry may also change the exchange coupling to a nearby impurity. ${ }^{17,18,24}$ Here, we look at the influence of the decreased asymmetry on the $N=9$ Kondo resonance.

Figure 3(a) shows the $N=9$ Coulomb diamond for the mentioned barrier gate voltage configuration with no external magnetic field applied. A clear dip in the overall peak at zero bias is visible with the dip extending into the direct tunneling region. The existence of the central dip in the peak suggests that a strong antiferromagnetic coupling between the spin on the quantum dot and a second spin suppresses the Kondo effect in this region. ${ }^{19}$ This system can be characterized by two low-energy scales, the Kondo temperature $k_{B} T_{K}$ given by the width Kondo resonance and the superexchange coupling $E_{x}$ given by the width of the dip. Taking half the splitting of the Kondo resonance as the superexchange coupling, we find $E_{x} \sim$ $80 \mu \mathrm{eV}{ }^{19}$

Figure 3(b) shows the differential conductance of the split Kondo resonance as a function of $V_{s d}$ for various in-plane magnetic fields. The splitting in energy changes relatively little for small fields, $B=0 \mathrm{mT}$ and $B= \pm 100 \mathrm{mT}$, while the splitting becomes larger for large fields, $B= \pm 1.5 \mathrm{~T}$, due to the Zeeman effect. A detailed plot of the total splitting in energy $\Delta E$ of the two peaks as a function of magnetic field is seen in Fig. 3(c). Qualitatively, the splitting first decreases, plateaus out, and then increases for fields larger. The red solid guide to the eye illustrates this flat-bottomed behavior. The smallest energy splitting is observed for $B=225 \mathrm{mT}$ [see also the traces in Fig. 3(b)], which roughly coincides with the onset of the plateau in the peak splitting $\Delta E$ in Fig. 3(c).

Interestingly, the zero-bias differential conductance shows a nonmonotonic behavior as a function of applied magnetic field, see Fig. 3(d), with a maximum conductance at $B_{\max }=$ $225 \mathrm{mT}$, marked by the black arrow in the figure, corresponding
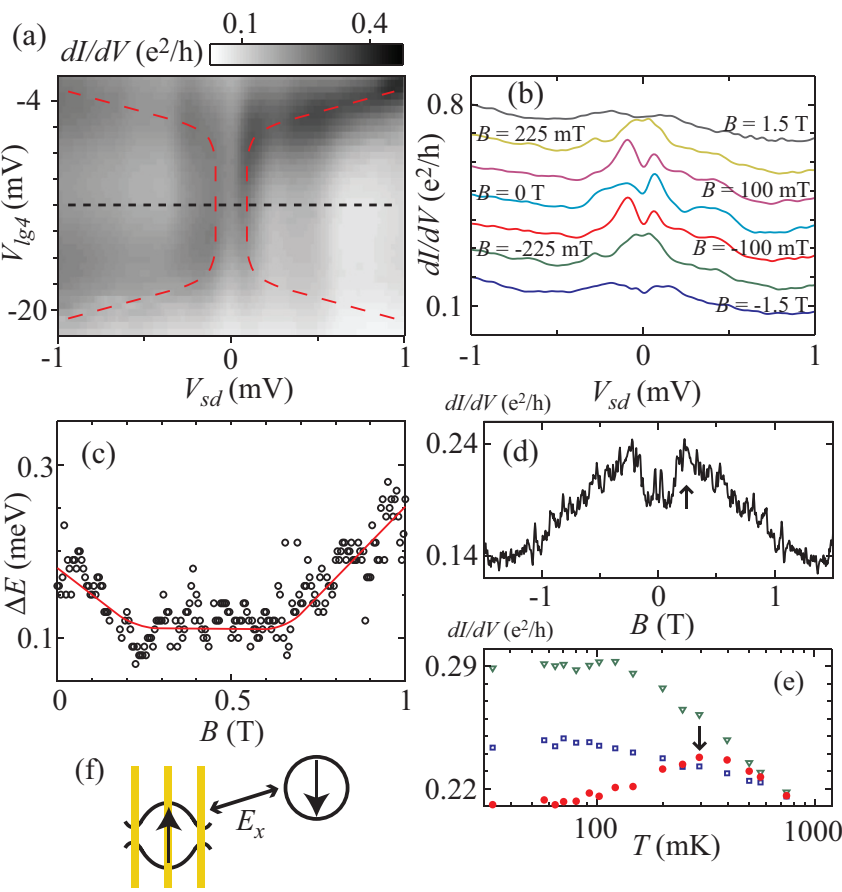

FIG. 3. (Color online) (a) Charge stability diagram of the $N=9$ Coulomb diamond for a decreased asymmetry of the barrier gate voltages showing a splitting of the zero-bias Kondo resonance for $B=0 \mathrm{~T}$. The differential conductance peaks have been outlined by dashed red lines for clarity. (b) Traces along the black dashed line in (a) for various magnetic fields. Each trace has a $0.1 e^{2} / \mathrm{h}$ offset for clarity. (c) Splitting of the Kondo resonance in energy as a function of magnetic field for $V_{l g 4}=-12 \mathrm{mV}$. A flat-bottomed red solid line has been included as a guide to the eye. (d) Differential conductance as a function of magnetic field for $V_{s d}=0 \mu \mathrm{V}$ and $V_{l g 4}=-12 \mathrm{mV}$. (e) Temperature dependence of the differential conductance of the split Kondo peak on a logarithmic temperature scale measured at $V_{s d}=80 \mu \mathrm{V}$ (triangles), $V_{s d}=-80 \mu \mathrm{V}$ (squares), and $V_{s d}=0 \mu \mathrm{V}$ (circles) for $V_{l g 4}=-11 \mathrm{mV}$ and $B=0 \mathrm{~T}$. (f) Schematic showing a quantum dot containing an electron spin and a nearby electron spin, localized to an impurity, interacting via an exchange energy $E_{x}$.

to a Zeeman energy of $\left|g_{5}^{*}\right| \mu_{B} B_{\max } \sim 30 \mu \mathrm{eV}$. Looking again at the differential conductance traces at $B= \pm 225 \mathrm{mT}$ in Fig. 3(b), which correspond to the peaks in the zero-bias conductance at positive and negative fields in Fig. 3(d), we clearly see that the splitting has decreased and the single Kondo resonance is almost completely restored. Following the procedure given in the previous section we estimate $T_{K} \sim$ $0.7 \mathrm{~K}$, corresponding to $k_{B} T_{K} \sim 60 \mu \mathrm{eV}$, from the width of the restored Kondo resonance at $B_{\max }$, i.e., in the region where the antiferromagnetic coupling is suppressed. Importantly, we find that $E_{x} \gtrsim k_{B} T_{K}$, which is consistent with theoretical predictions of two spins with antiferromagnetic coupling in the limit of $T \ll T_{K} .{ }^{19}$ A small residual splitting of the Kondo resonance is seen from $B \sim 225 \mathrm{mT}$ to $B \sim 650 \mathrm{mT}$ in Fig. 3(c). Theory predicts a complete restoration of the Kondo resonance when $\left|g_{5}^{*}\right| \mu_{B} B_{\max } \approx E_{x}$; see below. The origin of this residual splitting is unknown but it might be related to a dynamical effect of the relative strengths of the exchange coupling and the Zeeman energy of the two interacting spins. 
In order to verify the presence of Kondo correlation effects in the system, we investigate the temperature dependence of the differential conductance at three different source-drain voltages [see Fig. 3(e)], using $V_{l g 4}=-11 \mathrm{mV}$. For $V_{s d}=80 \mu \mathrm{V}$ (triangles) and $V_{s d}=-80 \mu \mathrm{V}$ (squares), which correspond to the two peaks at positive and negative bias voltages where the antiferromagnetic coupling is suppressed, we see typical behavior of a Kondo resonance with a logarithmic decrease of the conductance as a function of temperature. However, for $V_{s d}=0 \mu \mathrm{V}$ (circles) the temperature dependence is nonmonotonic, reaching a maximum conductance at $T_{\max } \sim 300 \mathrm{mK}$ (marked by the black arrow in the figure), corresponding to an energy of $k_{B} T_{\max } \sim 30 \mu \mathrm{eV}$. The energy scales of $T_{\max }$ and $B_{\max }$ are in quantitative agreement, which suggests that the same underlying physical mechanism gives rise to the observed phenomena.

A likely physical explanation of the observed scenario is that the gate-induced splitting of the Kondo resonance is caused by a crossover between two different phases, a conventional spin- $1 / 2$ Kondo phase and a two-impurity Kondo phase. In this interpretation, one of the two impurity spins is the spin localized to the quantum dot, whereas the other spin resides close to the quantum dot, possibly in a trap state in the $\mathrm{HfO}_{2} /$ semiconductor interface; see the schematic in Fig. 3(f). The two spins will interact via an exchange coupling $E_{x}$. In the weak-coupling regime (small $E_{x}$ ) the conventional Kondo effect dominates, leading to a zero-bias peak in the differential conductance. An increased coupling (large $E_{x}$ ) leads to a crossover to a different phase. The new phase is characterized by the formation of a spin-singlet state between the two interacting spins, i.e., an antiferromagnetic phase. The formation of the singlet state effectively locks the spin in the dot, thus suppressing the Kondo resonance around zero bias. For larger bias voltages, however, the system may occupy the higher-energy triplet state, leading to reestablishment of the Kondo resonance at the singlet-triplet degeneracy point. Our data are in agreement with these theoretical predictions. Furthermore, we find that the energy scales of the experimentally determined $T_{\max }, B_{\max }, E_{x}$, and $T_{K}$ are all of the same order of magnitude. Finally, we note that $E_{x} / k_{B} T_{K} \sim 1.3$ suggests that the system is in a crossover phase with asymmetric tunnel coupling and not at the quantum critical point where $E_{x} / k_{B} T_{K} \sim 2.5 .{ }^{19}$

\section{THEORETICAL MODEL}

In order to qualitatively describe the experimental results, we employ a two-site $(i=1$ dot site and $i=2$ impurity site) Hubbard model with a common on-site energy $\epsilon_{\sigma}$, and electron-electron repulsion $U$ which is connected to the left and right leads:

$$
\begin{aligned}
& \mathcal{H}_{d}=\sum_{i \sigma} \epsilon_{\sigma} d_{i \sigma}^{\dagger} d_{i \sigma}+\sum_{i} U n_{i \uparrow} n_{i \downarrow}+\sum_{\sigma} t d_{1 \sigma}^{\dagger} d_{2 \sigma}+\text { H.c. } \\
& \mathcal{H}_{C}=\sum_{k_{\alpha} \in\{L, R\}} E_{k_{\alpha}} c_{k_{\alpha}}^{\dagger} c_{k_{\alpha}}, \\
& \mathcal{H}_{T}=\sum_{k_{L} \sigma}\left(V_{L} c_{k_{L} \sigma} d_{1 \sigma}^{\dagger}+\text { H.c. }\right)+\sum_{k_{R} \sigma}\left(V_{R} c_{k_{R} \sigma} d_{2 \sigma}^{\dagger}+\text { H.c. }\right) .
\end{aligned}
$$

Nonequilibrium effects in $\mathcal{H}=\mathcal{H}_{d}+\mathcal{H}_{C}+\mathcal{H}_{T}$ are investigated by employing the finite- $U$ slave boson mean field formulation developed by Kotliar and Ruckenstein (KR $)^{36}$ which has been successfully applied in the strong-coupling limit. In this approach, the localized electron operator $d_{i \sigma}$ is replaced by a combination of a quasiparticle fermion operator $f_{i \sigma}$ and the empty $e_{i}$, double-occupancy $d_{i}$, and single-occupancy magnetic $p_{i \sigma}$ slave-boson operators. Thus, the four many-body localized states $|0\rangle,|\uparrow\rangle,|\downarrow\rangle$, and $|\uparrow \downarrow\rangle$ are defined as $e^{\dagger}=|0\rangle, p_{\uparrow}^{\dagger}=|\uparrow\rangle, p_{\downarrow}^{\dagger}=|\downarrow\rangle$, and $d^{\dagger}=|\uparrow \downarrow\rangle$, together with the closure relation

$$
\begin{array}{r}
|0\rangle\left\langle 0\left|+\sum_{\sigma}\right| \sigma\right\rangle\langle\sigma|+| \uparrow \downarrow\rangle\langle\uparrow \downarrow| \\
=e^{\dagger} e+\sum_{\sigma} p_{\sigma}^{\dagger} p_{\sigma}+d^{\dagger} d=1,
\end{array}
$$

and the charge conservation condition

$$
Q_{\sigma} \equiv f_{\sigma}^{\dagger} f_{\sigma}=p_{\sigma}^{\dagger} p_{\sigma}+d^{\dagger} d .
$$

In this representation the electron creation projector with spin $\sigma$ becomes $|\sigma\rangle\langle 0|+| \uparrow \downarrow\rangle\langle\bar{\sigma}|$, which is translated into $z_{\sigma}^{\dagger}=$ $p_{\sigma}^{\dagger} e+d^{\dagger} p_{\bar{\sigma}}$ (up to a normalization factor). ${ }^{36}$ The mean-field approach consists of replacing the slave-boson operators by their mean-field values $e_{i} \rightarrow\left\langle e_{i}\right\rangle, p_{i \sigma} \rightarrow\left\langle p_{i \sigma}\right\rangle$, and $d_{i} \rightarrow$ $\left\langle d_{i}\right\rangle$. By doing this, the new slave-boson Hamiltonian becomes quadratic in which the two constrictions are added as two Lagrange multipliers $\left(\lambda_{i}, \Lambda_{i}\right)$. The mean-field parameters are calculated with the set of equations derived from the equation of motion of the slave-boson operators and the two constraints. In this manner we obtain the renormalized parameters for the localized level positions and the tunneling amplitudes:

$$
\widetilde{\varepsilon}_{i \sigma} \rightarrow \varepsilon_{i \sigma}+\Lambda_{i \sigma}, \quad \tilde{t}_{\sigma}=t z_{1 \sigma}^{*} z_{2 \sigma}, \quad \widetilde{V}_{\alpha}=V_{\alpha} z_{\alpha \sigma} .
$$

The current through the dot is calculated from the time variation of the left lead occupation as $I=I_{L}=e \frac{d}{d t} \hat{N}_{L}=$ $\frac{i e}{\hbar}\left[\mathcal{H}, n_{L}\right]$, where $N_{L}=\sum_{k, \sigma} c_{L k \sigma}^{\dagger} c_{L k \sigma}$. Then, the current becomes

$$
\langle I\rangle=-\frac{e}{h} \int d \hbar \omega\left[f_{L}(\hbar \omega)-f_{R}(\hbar \omega)\right] T(\hbar \omega),
$$

where the transmission probability is defined as $T(\hbar \omega) \equiv$ $4 \sum_{\sigma} h \Gamma_{L} h \Gamma_{R} t^{2} \Delta^{-1}(\hbar \omega) \quad$ with $\quad \Delta(\hbar \omega) \equiv \chi(\hbar \omega) \chi^{*}(\hbar \omega)$, $\chi(\hbar \omega)=\left[\left(\hbar \omega-\epsilon_{\sigma}+i h \Gamma_{L}\right)\left(\hbar \omega-\epsilon_{\sigma}+i h \Gamma_{R \sigma}\right)-t^{2}\right], \quad$ and $h \Gamma_{L(R)}=\pi V_{L(R)}^{2} \rho_{L(R)}(\hbar \omega)$ as the left/right lead-dot hybridization energy [with $\rho_{L(R)}(\hbar \omega)=1 / D$ for $-D \leqslant \hbar \omega \leqslant D$, and $D$ being the contact bandwidth].

In the Kondo regime, Eq. (3) is equivalent to the twoimpurity Kondo Hamiltonian

$$
H_{K}=\sum_{i} J_{K} \vec{S}_{i} \vec{\sigma}_{i}+E_{x} \vec{S}_{1} \vec{S}_{2},
$$

where $S_{1}$ and $S_{2}$ are the spin operators for the localized spins, and $\sigma_{1(2)}$ is the local spin in the $i=1,2$ contact region $(1 \equiv L$, and $2 \equiv R$ ). For the sake of simplicity we consider the same Kondo strength $J_{K}$ for the dot and the magnetic impurity. $E_{x}=$ $4 t^{2} / U$ denotes the superexchange interaction between the dot and the impurity spins. The competition between the Kondo coupling and the superexchange occurs as a crossover between two phases, namely the Kondo phase where $E_{x} \ll k_{B} T_{K}$ and 

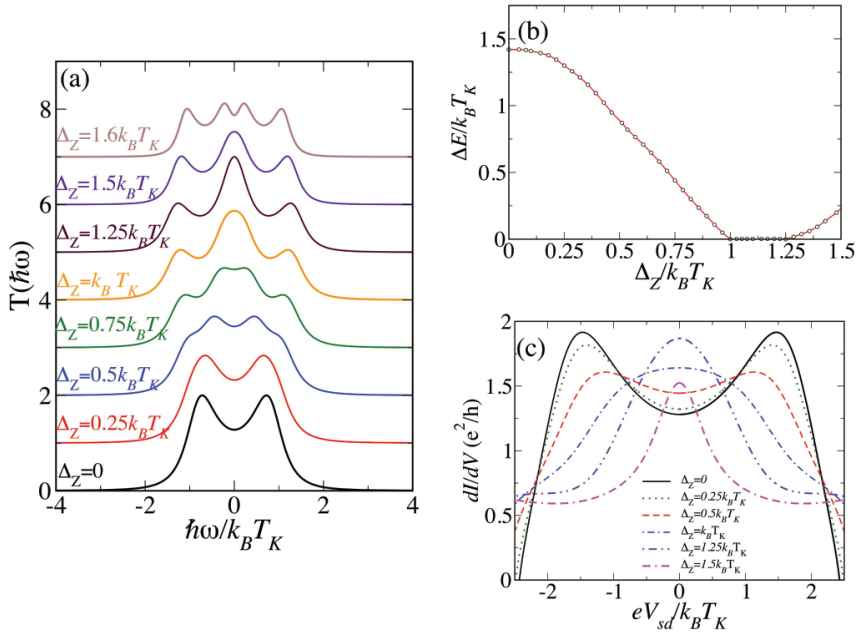

FIG. 4. (Color online) (a) Transmission $T(\hbar \omega)$ for different Zeeman energies $\Delta_{Z}=\left|g_{5}^{*}\right| \mu_{B} B$. (b) Evolution of the splitting of the two central peaks in the transmission $(\Delta E)$ versus $\Delta_{Z}$. (c) Differential conductance as a function of the external magnetic field. Parameters: $\epsilon=-U / 2, U=3 h \Gamma$, and $t=h \Gamma$. With the parameters $E_{x}=4 t^{2} / U \approx 1.25 h \Gamma, T_{K}(-U / 2)=0.53 h \Gamma$, the dot system is found in its antiferromagnetic phase for $B=0$.

the antiferromagnetic phase where the two localized spins are combined into a singlet state $\left(E_{x} \gg k_{B} T_{K}\right)$. When a magnetic field is applied, the Zeeman term is added to $\mathcal{H}$ :

$$
\mathcal{H}_{Z}=\left|g_{5}^{*}\right| \mu_{B} B \vec{S}_{1 z}+\left|g_{5}^{*}\right| \mu_{B} B \vec{S}_{2 z} .
$$

The presence of a magnetic field tends to align the two localized spins, $S_{z 1}=S_{z 2}$. From the point of view of the dot, the superexchange coupling $E_{x}$ acts as an effective magnetic field generated by the magnetic impurity $B_{\mathrm{exc}}=E_{x} S_{z 2}$. Therefore, when the external magnetic field compensates the exchange field the Kondo restoration occurs and the transmission through the quantum dot shows a Kondo peak. By further increasing the magnetic field the Kondo resonance splits again and eventually the Kondo effect is suppressed.

All these above-described features that are observed in the experiment are qualitatively reproduced by our numerical simulations shown in Fig. 4(a). The figure illustrates the magnetic field dependence of the transmission $T(\hbar \omega)$. At $B=$ 0 the transmission shows a double-peak structure with peaks at $\omega \approx \pm E_{x} / 2$ due to the strong superexchange interaction $\left(E_{x} \gtrsim k_{B} T_{K}\right)$. By increasing $B$ one clearly observes that the splitting of $T(\hbar \omega)$ is destroyed and a resonance at the Fermi energy emerges restoring the Kondo effect around some critical magnetic field $B_{c}$. By further increasing $B$ the Kondo resonance splits again. In general for $B, E_{x}$, and $T_{K}$ we find four peaks in the $T(\hbar \omega)$ corresponding to the possible states of the two antiferromagnetically coupled spin- $1 / 2$ impurities in a magnetic field. The restoration of the Kondo resonance takes place at the critical value $g_{5}^{*} \mu_{B} B_{c} \approx E_{x}$ where the two peaks merge together and give a central Kondo resonance. The value of $B_{c}$ corresponds to the special point where the antiferromagnetic singlet state formed by the dot spin and the magnetic impurity and the lowest component of the triplet state become degenerate. The evolution of the splitting $\Delta E$ in the transmission is shown in Fig. 4(b) that nicely agrees with the experimental finding shown in Fig. 3(c). Finally, Fig. 4(c) shows the nonlinear conductance when the Kondo restoration occurs in qualitative agreement with the experimental result of Fig. 3(b). In conclusion, these calculations support the physical interpretation of the experimental features in terms of magnetic-field-induced Kondo restoration within the twoimpurity Kondo effect scenario.

\section{DISCUSSION AND CONCLUSIONS}

Zero-field splittings of Kondo resonances stemming from an unintentional two-impurity Kondo effect have been experimentally observed in carbon nanotubes. ${ }^{22-24}$ Unlike Refs. 22 and 23, we have observed a gate-induced crossover and unlike Ref. 24 the temperature dependence of our data shows that the observed behavior indeed stems from Kondo correlation effects. A zero-field splitting of a Kondo resonance has also been observed in an even-occupation quantum $\operatorname{dot}^{27}$ with a total spin $S=1$ stemming from the two-stage Kondo effect. $^{28,29}$ While the two-impurity and two-stage Kondo effects manifest themselves in a similar way, we believe that the two-stage Kondo effect is an unlikely physical explanation of our observations. The two-stage Kondo effect requires a higher spin state, i.e., $S>1 / 2$, which is unlikely in our $N=9$ quantum dot system as this would require a triple orbital degeneracy or near degeneracy with $S=3 / 2$.

In summary, we have demonstrated a gate-induced crossover between a spin- $1 / 2$ Kondo phase and a phase which shows a splitting of the Kondo resonance in a quantum dot at zero magnetic field. We interpret the observed zero-field splitting of the Kondo resonance in terms of the two-impurity Kondo effect where the crossover is induced by a gate tunable exchange interaction between the dot electron and a localized impurity electron. We find qualitative agreement between experimental and theoretical results for the magnetic field dependence of the split resonance. We hope that our analysis of the unintentional two-impurity Kondo effect in the interacting and noninteracting regions in both the linear and nonlinear response regimes will help shed new light on the nature of the interactions between spins in quantum dots and impurities in the environment. Controlling or reducing unwanted interactions with the environment is of utmost importance for the realization of spintronic and quantum computational devices.

\section{ACKNOWLEDGMENTS}

We thank A. Oiwa for useful discussions. M.L. and H.Q.X. were supported by the Swedish Research Council (VR). R.L. and J.S.L. were supported by MICINN Grants No. FIS201123526 and No. CSD2007-00042 (CPAN). H.Q.X. also acknowledges financial support from the National Basic Research Program of the Ministry of Science and Technology of China (Grants No. 2012 CB932703 and No. 2012 CB932700) and from the National Natural Science Foundation of China (Grant No. 91221202). 
*Present address: Department of Applied Physics, The University of Tokyo, 7-3-1 Hongo, Bunkyo-ku, Tokyo 113-8656, Japan; marcus@meso.t.u-tokyo.ac.jp

${ }^{1}$ R. Hanson, L. P. Kouwenhoven, J. R. Petta, S. Tarucha, and L. M. K. Vandersypen, Rev. Mod. Phys. 79, 1217 (2007).

${ }^{2}$ L. I. Glazman and M. E. Raikh, Pis'ma Zh. Eksp. Teor. Fiz. 47, 378 (1988) [JETP Lett. 47, 452 (1988)].

${ }^{3}$ T. K. Ng and P. A. Lee, Phys. Rev. Lett. 61, 1768 (1988).

${ }^{4}$ D. Goldhaber-Gordon, H. Shtrikman, D. Mahalu, D. AbuschMagder, U. Meirav, and M. A. Kastner, Nature (London) 391, 156 (1998).

${ }^{5}$ S. M. Cronenwett, T. H. Oosterkamp, and L. P. Kouwenhoven, Science 281, 540 (1998).

${ }^{6}$ J. Nygård, D. H. Cobden, and P. E. Lindelof, Nature (London) 408, 342 (2000).

${ }^{7}$ Y. Igarashi, M. Jung, M. Yamamoto, A. Oiwa, T. Machida, K. Hirakawa, and S. Tarucha, Phys. Rev. B 76, 081303(R) (2007).

${ }^{8}$ J. Park, A. N. Pasupathy, J. I. Goldsmith, C. Chang, Y. Yaish, J. R. Petta, M. Rinkowski, J. P. Sethna, H. D. Abruña, P. L. McEuen, and D. C. Ralph, Nature (London) 417, 722 (2002).

${ }^{9}$ A. Fuhrer, T. Ihn, K. Ensslin, W. Wegscheider, and M. Bichler, Phys. Rev. Lett. 93, 176803 (2004).

${ }^{10}$ T. S. Jespersen, M. Aagesen, C. Sørensen, P. E. Lindelof, and J. Nygård, Phys. Rev. B 74, 233304 (2006).

${ }^{11}$ H. A. Nilsson, P. Caroff, C. Thelander, M. Larsson, J. B. Wagner, L.-E. Wernersson, L. Samuelson, and H. Q. Xu, Nano Lett. 9, 3151 (2009).

${ }^{12}$ W. G. van der Wiel, S. De Franceschi, J. M. Elzerman, S. Tarucha, L. P. Kouwenhoven, J. Motohisa, F. Nakajima, and T. Fukui, Phys. Rev. Lett. 88, 126803 (2002).

${ }^{13}$ S. Sasaki, S. De Franceschi, J. M. Elzerman, W. G. van der Wiel, M. Eto, S. Tarucha, and L. P. Kouwenhoven, Nature (London) 405, 764 (2000).

${ }^{14}$ H. A. Nilsson, O. Karlström, M. Larsson, P. Caroff, J. N. Pedersen, L. Samuelson, A. Wacker, L.-E. Wernersson, and H. Q. Xu, Phys. Rev. Lett. 104, 186804 (2010).

${ }^{15}$ P. Jarillo-Herrero, J. Kong, H. S. J. van der Zant, C. Dekker, L. P. Kouwenhoven, and S. De Francenschi, Nature (London) 434, 484 (2005)
${ }^{16}$ R. M. Potok, I. G. Rau, H. Shtrikman, Y. Oreg, and D. GoldhaberGordon, Nature (London) 446, 167 (2007).

${ }^{17}$ H. Jeong, A. M. Chang, and M. R. Melloch, Science 293, 2221 (2001).

${ }^{18}$ N. J. Craig, J. M. Taylor, E. A. Lester, C. M. Marcus, M. P. Hanson, and A. C. Gossard, Science 304, 565 (2004).

${ }^{19}$ P. Simon, R. López, and Y. Oreg, Phys. Rev. Lett. 94, 086602 (2005).

${ }^{20}$ C. H. Chung and W. Hofstetter, Phys. Rev. B 76, 045329 (2007).

${ }^{21}$ H. B. Heersche, Z. de Groot, J. A. Folk, L. P. Kouwenhoven, H. S. J. van der Zant, A. A. Houck, J. Labaziewicz, and I. L. Chuang, Phys. Rev. Lett. 96, 017205 (2006).

${ }^{22}$ J. Nygård, W. F. Koehl, N. Mason, L. DiCarlo, and C. M. Marcus, arXiv:cond-mat/0410467.

${ }^{23}$ Y. Bomze, I. Borzenets, H. Mebrahtu, A. Makarovski, H. U. Baranger, and G. Finkelstein, Phys. Rev. B 82, 161411 (2010).

${ }^{24}$ A. Eichler, M. Weiss, and C. Schönenberger, Nanotechnology 22, 265204 (2011)

${ }^{25}$ M. A. Ruderman and C. Kittel, Phys. Rev. 96, 99 (1954).

${ }^{26}$ K. Yosida, Phys. Rev. 106, 893 (1957).

${ }^{27}$ G. Granger, M. A. Kastner, I. Radu, M. P. Hanson, and A. C. Gossard, Phys. Rev. B 72, 165309 (2005).

${ }^{28}$ M. Pustilnik and L. I. Glazman, Phys. Rev. Lett. 87, 216601 (2001).

${ }^{29}$ W. Hofstetter and H. Schoeller, Phys. Rev. Lett. 88, 016803 (2001); W. Hofstetter and G. Zarand, Phys. Rev. B 69, 235301 (2004).

${ }^{30}$ M. Larsson and H. Q. Xu, Phys. Rev. B 83, 235302 (2011).

${ }^{31}$ A. V. Kretinin, H. Shtrikman, and D. Mahalu, Phys. Rev. B 85, 201301 (2012)

${ }^{32}$ F. D. M. Haldane, Phys. Rev. Lett. 40, 416 (1978).

${ }^{33}$ G. Breit and E. Wigner, Phys. Rev. 49, 519 (1936).

${ }^{34}$ M. Larsson, H. A. Nilsson, H. Hardtdegen, and H. Q. Xu, Appl. Phys. Lett. 95, 192112 (2009).

${ }^{35}$ D. Goldhaber-Gordon, J. Göres, M. A. Kastner, H. Shtrikman, D. Mahalu, and U. Meirav, Phys. Rev. Lett. 81, 5225 (1998).

${ }^{36}$ G. Kotliar and A. E. Ruckenstein, Phys. Rev. Lett. 57, 1362 (1986). 